\title{
Detecting microstructural deviations in individuals with deep diffusion MRI tractometry
}

\author{
Maxime Chamberland ${ }^{1,2}{ }^{凶}$, Sila Genc ${ }^{1}$, Chantal M. W. Tax ${ }^{1,3}$, Dmitri Shastin ${ }^{1,4}{ }^{1,}$, Kristin Koller ${ }^{1}$, \\ Erika P. Raven ${ }^{1,5}$, Adam Cunningham ${ }^{6}$, Joanne Doherty ${ }^{1,6}$, Marianne B. M. van den Bree ${ }^{6}$, \\ Greg D. Parker ${ }^{1}$, Khalid Hamandi ${ }^{1,4,7}$, William P. Gray ${ }^{1,4,7}$ and Derek K. Jones ${ }^{1}$
}

\begin{abstract}
Most diffusion magnetic resonance imaging studies of disease rely on statistical comparisons between large groups of patients and healthy participants to infer altered tissue states in the brain; however, clinical heterogeneity can greatly challenge their discriminative power. There is currently an unmet need to move away from the current approach of group-wise comparisons to methods with the sensitivity to detect altered tissue states at the individual level. This would ultimately enable the early detection and interpretation of microstructural abnormalities in individual patients, an important step towards personalized medicine in translational imaging. To this end, Detect was developed to advance diffusion magnetic resonance imaging tractometry towards single-patient analysis. By operating on the manifold of white-matter pathways and learning normative microstructural features, our framework captures idiosyncrasies in patterns along white-matter pathways. Our approach paves the way from traditional group-based comparisons to true personalized radiology, taking microstructural imaging from the bench to the bedside.
\end{abstract}

$\mathrm{N}$ ormative modeling is an emerging statistical framework that aims to capture variability by comparing individuals with a normative population ${ }^{1}$. The benefits of normative modeling in neuroimaging in general are well documented, with applications in atypical brain development and psychiatry (see ref. for a review). Applications of deep normative modeling have mostly been demonstrated at the voxel level using functional MRI data ${ }^{3}$ and volumetric data derived from standard structural magnetic resonance imaging $(\mathrm{MRI})^{4,5}$

Diffusion MRI (dMRI) is another MRI modality that allows non-invasive characterization of tissue microstructure. In the brain, for example, information about the structural architecture of the white matter can be obtained by probing the random motion of water molecules ${ }^{6}$ and acquiring multiple magnetic resonance images with different diffusion-senitization properties. The ability to derive semiquantitative features such as fractional anisotropy or mean diffusivity ${ }^{8}$, or to virtually reconstruct white-matter pathways with tractography ${ }^{9}$ has had a huge impact on the ability to distinguish between typical and atypical brain structures in vivo in health and diseas $\mathrm{e}^{10}$; however, prediction modeling (or case-control differentiation), in which a group of $N$ patients with the same disease is compared with a group of $N$-matched controls, is not well suited to clinically heterogeneous groups (for example, neurological disorders ${ }^{11}$, psychiatric disorders such as schizophrenia or autism spectrum disorder, and rare cases). Despite decades of progress in the research domain, the primary clinical use of dMRI has been for largely limited to diagnosing acute ischemic stroke or grading and monitoring of tumor invasion. Several studies have shown success in identifying subtle but important microstructural changes at the individual patient level ${ }^{12}$, showcasing the potential of dMRI to be applied more broadly across applications. Yet there is a scarcity of dMRI frameworks for single-patient analysis (that is, one patient versus $N$ controls). There is an urgent yet unmet need for a paradigm shift from group-wise comparisons to individualized diagnosis, that is, detecting whether (and where) the tissue microstructure of a single participant is abnormal ${ }^{13}$; not only would this greatly facilitate the study of clinically heterogeneous groups ${ }^{1,4}$, it would also facilitate the study of rare diseases and true clinical adoption (that is, making a diagnosis/prognosis in an individual patient).

As mentioned above, current efforts to apply deep normative modeling to neuroimaging have so far relied on voxel-based methods. For the assessment of white matter-which comprises continuous pathways - under conditions in which an entire pathway may be affected (for example, in developmental disorders such as schizophrenia ${ }^{14}$ ), fragmenting the analysis in this way is suboptimal, whereas operating on the manifold of reconstructed tracts offers a more intuitive approach. Indeed, in comparing microstructural properties between groups, many computational pipelines adopt a tractometry approach ${ }^{15,16}$, that is, mapping measures along pathways reconstructed via tractography, either by averaging along the whole $\operatorname{tract}^{17}$ or a segment thereof ${ }^{18-21}$. Along-tract profiling has been applied previously to investigate various brain conditions ${ }^{15,19,22-24}$. The main advantage here is that image registration can be avoided as tractometry is performed in each patient's native space, resulting in a set of individual tract profiles. There are, however, some limitations. First, most analyses treat tractometry measures from specific pathways as independent measures. This univariate approach has the potential to obscure key relationships between different tracts.

${ }^{1}$ Cardiff University Brain Research Imaging Centre (CUBRIC), School of Psychology, Cardiff University, Cardiff, UK. ${ }^{2}$ Donders Institute for Brain, Cognition and Behavior, Radboud University, Nijmegen, the Netherlands. ${ }^{3}$ Image Sciences Institute, University Medical Center Utrecht, Utrecht, the Netherlands. ${ }^{4}$ Department of Neuroscience, University Hospital of Wales (UHW), Cardiff, UK. ${ }^{5}$ Bernard and Irene Schwartz Center for Biomedical Imaging, Department of Radiology, New York, NY, USA. ${ }^{6}$ Medical Research Council Centre for Neuropsychiatric Genetics and Genomics, Division of Psychological Medicine and Clinical Neurosciences, Cardiff University, Cardiff, UK. ${ }^{7}$ Brain Repair and Intracranial Neurotherapeutics (BRAIN) Unit, School of Medicine, Cardiff University, Cardiff, UK.凶e-mail: maxime.chamberland@donders.ru.nl 


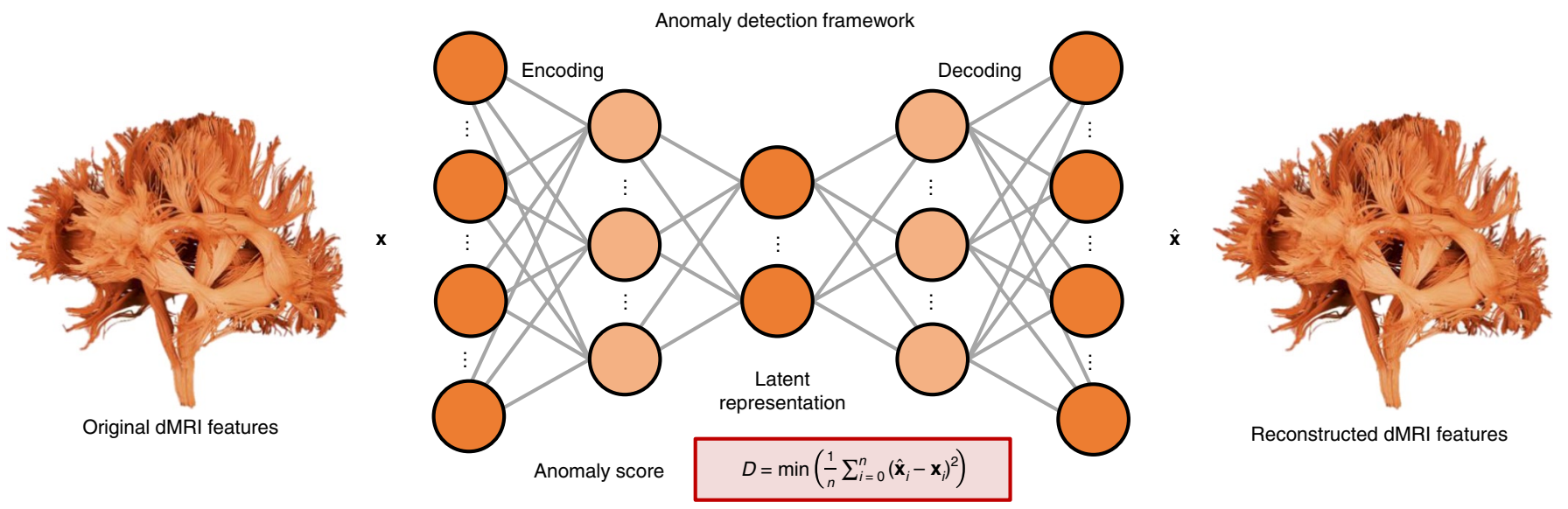

Fig. 1 | Graphical representation of the proposed anomaly detection framework. The neural network consists of a deep autoencoder symmetrically designed with five fully connected layers. The input and output layers have exactly the same number of nodes as the number of input tracts features (|x|, represented by the colored vector). The goal of the network is to generate an output ( $\hat{\mathbf{x}})$ similar to the input (x) by minimizing the reconstruction error $(D)$. Here, the mean absolute error (MAE) was used as the anomaly score; MAE measures the average magnitude of the errors and is derived during testing by computing the absolute differences between the reconstructed microstructural features $\left(\hat{\mathbf{x}}_{i}\right)$ and the raw input features $\left(\mathbf{x}_{i}\right)$. The number of input features is denoted by $n$, with the current feature denoted by $i$. Node coloring is for illustrative purposes only.

Focusing on any particular anatomical location therefore increases the risk of losing the full picture. Although individual pathways can appear normal in isolation, by considering them as an ensemble (for example, as part of a network), any such intertract relationships could collectively help to identify outliers. Second, when analyzing multiple measures (even when derived within the same tract), statistical analysis is hampered by: (1) the multiple comparisons problem; and (2) any covariance between measurements ${ }^{17,25}$. Here multidimensional approaches can increase statistical power by combining the sensitivity profiles of independent modalities ${ }^{25-27}$.

Here we present an anomaly detection framework (Detect) that uses a data-driven, unsupervised normative modeling approach based on deep autoencoders ${ }^{28}$. Autoencoders are a type of artificial neural network traditionally used for dimensionality reduc$\operatorname{tion}^{28}$. They can capture non-linear interactions between the input features by learning a self-representation of their inputs through a low-dimensional layer (Fig. 1); the goal is to generate an output $(\hat{\mathbf{x}})$ similar to the input $(\mathbf{x})$ by minimizing the reconstruction error. This same representation can be exploited for anomaly detection by analyzing deviations in the reconstruction. Detect moves substantially beyond dMRI group-level analysis techniques by identifying and localizing anomalies in multiple tract profiles at once at the individual level. The proposed framework was trained to learn normative sets of features derived from healthy brain tract profiles in three independent datasets and one reproducibility datase ${ }^{29}$. Once trained, the network is then presented with unseen healthy tract profiles (for testing) and subsequently exposed to tract profiles from individuals diagnosed with neurological/psychiatric conditions. We emphasize that the model has no access to the diagnostic labels during the training phase and thus our anomaly detection approach is fully unsupervised. To assess generalization, the framework was then applied to single participants with a range of neurological and psychiatric disorders, including children and adolescents with copy number variants (CNVs) at high risk of neurodevelopmental and psychiatric disorders; patients with drug refractory epilepsy; and patients with schizophrenia (SCHZ). We compared the performance of our approach with (1) a conventional $z$-score-distribution approach and (2) principal component analysis (PCA) combined with the Mahalanobis distance (a widely used approach in cluster analysis and classification techniques) ${ }^{21,24,27}$. Importantly, the analyses of the three pathological cases were performed in the browser, from the point-of-view of future Detect users.

\section{Results}

Framework overview. Detect is an open-source, user-facing tool built on the interactive Streamlit framework, which promotes data exploration through interactive visualizations in the browser. The framework offers three main scripts: Detect, Inspect and Relate. Detect facilitates patient comparisons using cross-validated area under the curve (AUC) computed over a set of user-defined iterations, by means of $z$-score, PCA or autoencoder (see Supplementary Fig. 4). The output is a single, bootstrapped anomaly score for each patient. Inspect allows the user to select a single subject and to compare it with the rest of the population. Here, feature anomalies are highlighted using a leave-one-out cross-validation approach, and only segments along the tract where two consecutive outliers occurred are reported. Results are displayed in real-time during each iteration for the user to evaluate. Furthermore, both scripts allow the visualization of tract profiles. Finally, Relate is a simple visual interface for correlating the anomaly scores obtained by the previous commands with clinical scores. In all scenarios, microstructural features included fractional anisotropy, mean diffusivity and rotationally invariant spherical harmonic features of zeroth and second orders ${ }^{30}$ (RISH0 and RISH2, respectively) derived from the largest $b$-value data in each dataset to maximize sensitivity to the intra-axonal signal ${ }^{31}$.

White-matter anomaly detection in CNV participants. Discriminating power. First we investigate individual differences in white-matter microstructure in children with CNVs at high genetic risk of neurodevelopmental and psychiatric disorders ${ }^{32}$, which are relatively rare and therefore challenging to recruit for research imaging studies ${ }^{33}$. Note that the framework was trained using data from typically developing children only. In general, the autoencoder approach showed higher AUC scores across the microstructural metrics and was better at identifying CNV patients as outliers, providing substantially higher sensitivity-specificity tradeoffs than the $z$-score and Mahalanobis-based approaches (Supplementary Fig. 1 and Supplementary Table 2). For example, the RISH0 feature showed higher discriminating power $(\mathrm{AUC}=0.83 \pm 0.08) \mathrm{com}-$ pared with the mean univariate $z$-score $(A U C=0.80 \pm 0.09)$ and multivariate Mahalanobis distance $(\mathrm{AUC}=0.61 \pm 0.09)$. In comparing the RISH0 group distributions (Fig. 2), anomaly scores derived via the autoencoder were significantly different (KolmogorovSmirnov test $=0.62, P<0.003$; Cohen's $d$ effect size $=1.39$ ) between 


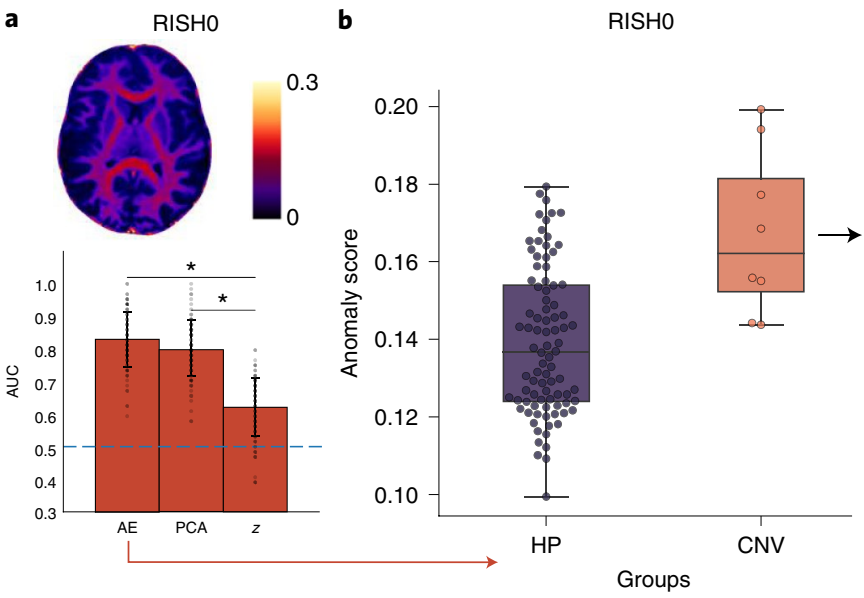

c

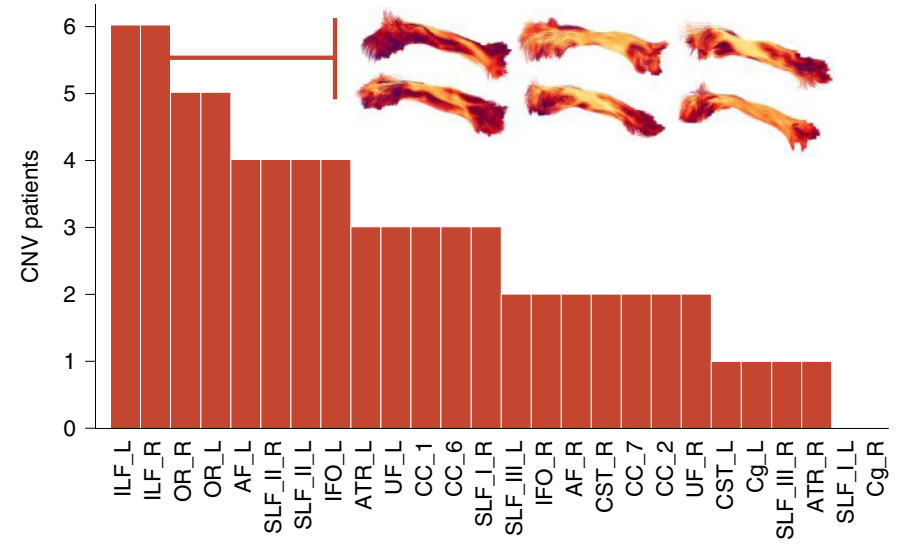

Bundles

Fig. 2 | Anomaly scores for the CNV dataset. a, The autoencoder (AE) network and PCA approaches provided better discriminating power in terms of sensitivity/specificity tradeoffs compared with traditional linear univariate approaches $\left({ }^{\star} P=5.69 \times 10^{-40}\right.$ and $P=1.92 \times 10^{-33}$, Bonferroni corrected with $\alpha=0.01$, two-tailed $t$-tests for AE- $z$ and PCA- $z$, respectively). The average AUC over 100 iterations (bottom) for the RISHO feature (top) is displayed (data are presented as mean values \pm s.d.). b. The RISHO features show higher reconstruction error for the CNV (orange box plot) compared with the typically developing patients (purple box plot) with a precision-recall AUC of 0.45 (center line, median; box limits, upper and lower quartiles; whiskers, 1.5 interquartile range; $n=90$ healthy participants and $n=8 \mathrm{CNVs}$ ). In comparison, a random classifier would score 0.08 . The box shows the quartiles of the dataset whereas the whiskers extend to show the rest of the distribution. $\mathbf{c}$, From a group perspective, anomaly rates were mostly observed in the ILF (color map: RISHO, lateral view), optic radiations and SLF. OR, optic radiations; UF, uncinate fasciculus; AF, arcuate fasciculus; IFO, inferior fronto-occipital fasciculus; CC, corpus callosum; $\mathrm{Cg}$, cingulum; ATR, anterior thalamic radiation, $R$, right brain hemisphere; $L$, left brain hemisphere.

the CNV individuals and the typically developing patients. In particular, all CNV patients had an anomaly score larger than the typically developing patient mean and $50 \%$ of them were larger than the 95th percentile of the typically developing patient population. In comparison, the difference between the anomaly scores was less pronounced with the $z$-score (Kolmogorov-Smirnov $=0.34, P=0.3$, Cohen's $d=0.38$ ) approaches, whereas similar results were obtained by using the Mahalanobis distance (Kolmogorov-Smirnov $=0.56$, $P=0.01$, Cohen's $d=1.20$ ).

Tract-specific deviations. A key advantage of using deep autoencoders for anomaly detection over traditional PCA-derived approach is its unique ability to relate the anomaly back to the individual elements of the input data. More specifically, the predicted data retains the same dimensionality as the input data and thus it is possible to see which feature cannot be accurately recovered by the autoencoder. For example, if a feature has a positive reconstruction error, then one can infer that the network learned a smaller value for that feature than what was provided as input. In the context of the CNV participants, multiple regions were highlighted (by positive reconstruction errors) as deviants from the typically developing patient population. Figure 3 reveals a high anomaly rate for various association bundles such as the bilateral inferior longitudinal fasciculus (ILF), optic radiations and the left superior longitudinal fasciculus (SLF_II). This is in line with current literature where microstructural differences are expected along association pathways, in agreement with psychotic symptoms ${ }^{34}$.

White-matter anomaly detection in epilepsy. Focal cortical dysplasia (FCD) - a malformation of cortical development-is the most common etiology in drug-resistant neocortical partial epilepsies $^{35}$. Although complete resection is the main predictor of seizure freedom following surgery, a substantial proportion of FCDs may be missed with standard clinical imaging protocols ${ }^{35}$ and the seizure-generating network may extend far beyond the visible dysplasia. Diffusion MRI contrast enhances the sensitivity of MRI to differences in the brain, but has only been reported at the group level ${ }^{36}$. Here we demonstrate two key advantages of the deep autoencoder approach in a clinical context. First, it succeeded in detecting white-matter anomalies that a conventional $z$-score-based approach has missed, potentially due to hidden interactions between the features. Second, detection of abnormal microstructural features away from putative seizure onset zone, as demonstrated in the first example, may contribute to the mapping of epileptogenic networks in individuals; thus, although the examples shown here had radiological changes detectable with T2-weighted sequences, the method could potentially be extended to cases of MRI-negative partial epilepsy increasing the diagnostic yield.

Patient 1 is a young adult female with seizures described as a fuzzy painful sensation in the torso rising up to the head, associated with mumbling sounds, occurring 2-5 times per day. A scalp video electroencephalogram (EEG) showed left temporal interictal epileptiform discharges and left temporal EEG onset. Clinical imaging demonstrated a small area of cortical-white-matter junction blurring in the laterobasal left temporal lobe associated with a transmantle area of T2 hyperintensity, suggestive of FCD type $\mathrm{II}^{37}$. Neuropsychological assessment was concordant with a left temporal deficit, also revealing preserved mesial structures manifesting in relatively preserved verbal memory performance. Subsequent stereo-EEG (SEEG) implantation confirmed ictal onset and prominent interictal discharges from neocortical contacts immediately behind the MRI lesion; furthermore, neocortical discharges were seen in SEEG contacts close to the temporal pole. Based on those clinical findings (neuropsychological assessment and SEEG), the patient proceeded to have resection with histology consistent with FCD. Five tracts of possible relevance were interrogated (Fig. 4). Microstructural anomalies were identified along the left ILF and optic radiations in the immediate proximity of the T2-weighted changes corresponding to SEEG contacts with maximal ictal EEG changes. Anomalies in the temporal portions of the left inferior fronto-occipital fasciculus and uncinate fasciculus pointed towards the temporal pole 



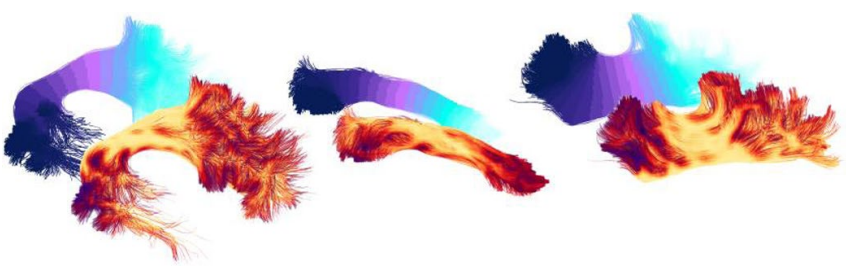

- AF

- ILF

- SLF

Fig. 3 | Along-tract anomalies in a single patient. Reconstructed tract profiles of CNV (top) and typically developing (bottom) patients reveals RISHO discrepancies along various association bundles such as the AF, ILF and SLF. The marked pink areas represent sections along the bundle where anomalies are detected by the leave-one-out cross-validation approach (threshold = 1 per number of healthy participants). Specifically, the autoencoder is trained using healthy participant data only, and compared with the FCD patient. Then for each of the healthy participants, the FCD patient is shuffled back in the population and the newly left out healthy participant is compared with that population. We then assess the MAE. The letters correspond to the tract list found in the Supplementary Information.

a
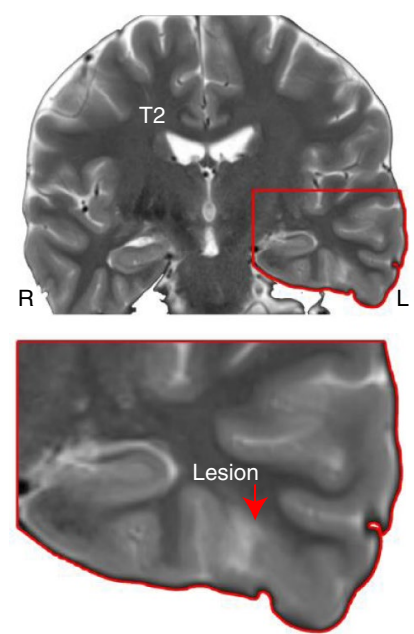
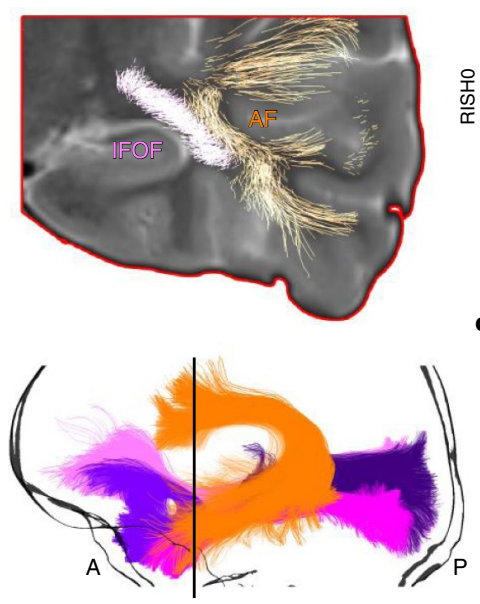

b

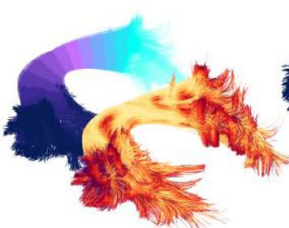

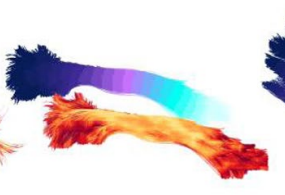

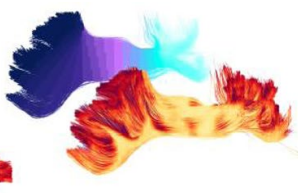

Fig. 4 | Focal cortical dysplasia anomaly detection (patient 1). a, The T2 hyperintense lesion, located at the base of the skull in the temporal lobe, is shown. b-d, Several pathways with anomalies interdigitate in the vicinity of the lesion. Although the inferior fronto-occipital fasciculus (c; IFOF with RISHO colormap overlayed and the 20 along-tract sections underlayed) signal did not extend beyond the shaded areas (d; $\pm 1 z$-score), the proposed anomaly detection framework identified abnormalities in that region (b, pink shaded areas; bold orange line, original tract-profiles; dotted purple line, reconstructed representation learnt from the network). $R$, right side; $L$, left side; $A$, anterior; $P$, posterior.

corroborating the SEEG findings that, despite normal clinical MRI, this area was part of the seizure network.

Patient 2 is an adult female with focal onset seizures since childhood occurring daily with episodes of loss of contact, grimacing and limb stiffening hypermotor movements, including clutching at nearby object on the left side. Scalp video EEG findings were consistent with frontal onset seizure semiology. Clinical MRI showed blurring of the cortical-white-matter junction between the right posterior superior frontal gyrus and the adjacent precentral gyrus, and a transmantle sign on T2/FLAIR from the cortex reaching all the way to the lateral ventricle, consistent with FCD type II. Subsequent SEEG recordings demonstrated spatial overlap between primary motor areas and early ictal onset and hence the patient did not proceed to surgery. Five tracts of possible relevance were interrogated with our framework (Fig. 5). Anomalies were detected corresponding to radiological and electrophysiological findings along the right corticospinal tract (CST), primary motor (CC4) and superior longitudinal fasciculus (SLF-I) beyond the visible lesion. No anomalies were found along the right cingulum and primary sensorimotor (CC5) regions.

The results are promising, with the tool identifying anomalies in concordance with clinical hypothesis in a single-patient analysis paradigm, testifying to its utility for clinical evaluation. Its extra value is highlighted by its sensitivity to outlying tract segments not detected with the conventional $z$-score approach. The $N=1$ approach to detect deep white-matter anomalies illustrated here will 

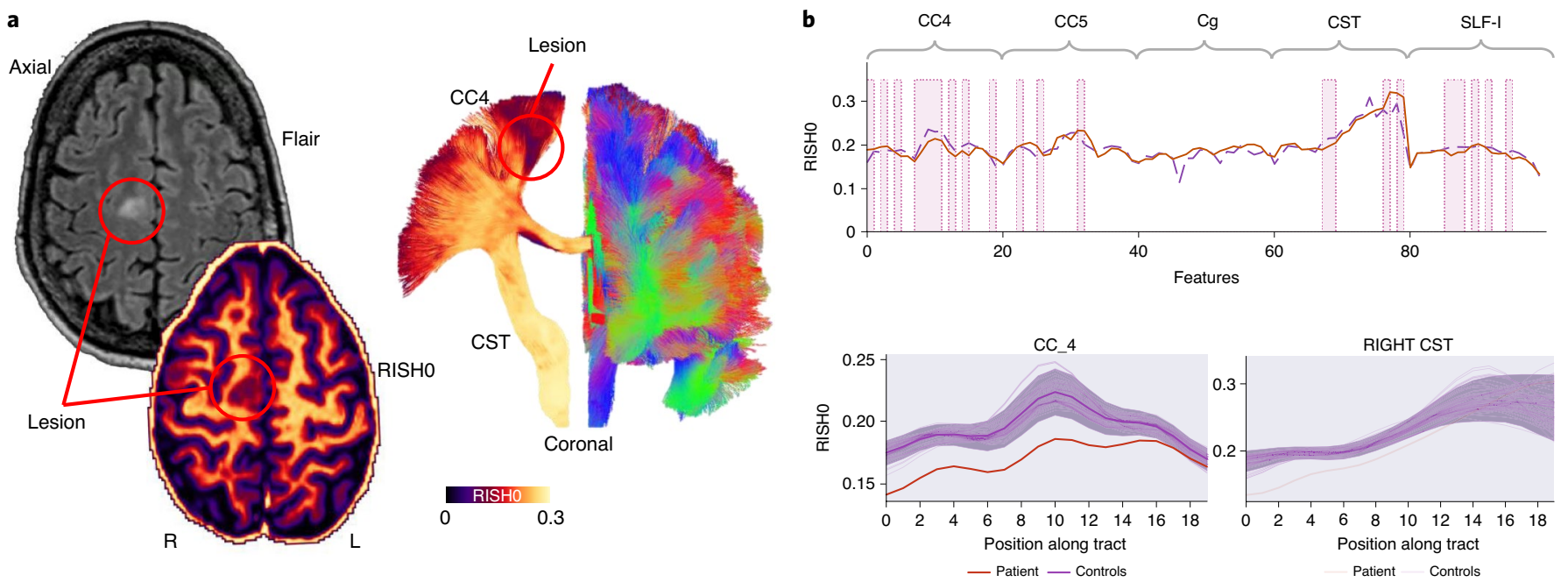

Fig. $\mathbf{5}$ | FCD anomaly detection (patient 2). $\mathbf{a}$, The lesion is located anterior to the right primary motor cortex in the supplementary motor area (hyperintense signal on the FLAIR image, hypointense on the RISH map). Tractography show tracts traversing the area (CC4). $\mathbf{b}$, Anomalies were identified in the right CC4, CST and SLF-I bundles (top, pink shaded areas). The bold orange line represents the original tract-profiles whereas the dotted purple line represents the reconstructed representation learned from the network. The $z$-score approach shows less focused anomaly patterns along the tracts (shaded area $= \pm 1 z$-score).

facilitate the identification of individualized therapy most appropriate to that patient, suggesting additional targets for diagnostic evaluation and possible surgical treatment.

Linking brain heterogeneity with epidemiological findings in schizophrenia. The extent to which individual clinical variability in schizophrenia relates to microstructural variability remains a key challenge in neuropsychiatry ${ }^{38}$, with most findings being at the group or voxel level. For the RISH0 feature, the autoencoder approach was better at identifying single $\mathrm{SCHZ}$ patients as outliers $(\mathrm{AUC}=0.64 \pm 0.06)$ when compared with PCA $(\mathrm{AUC}=0.59 \pm 0.07)$ or the $z$-score (AUC $=0.39 \pm 0.06$ ). In comparing these group distributions, anomaly scores derived from the autoencoder were found to be significantly different $(t=-2.60, P=0.01$, Cohen's $d=0.47)$ between the SCHZ individuals and the healthy participants (Fig. 6). In particular, 31 of the $43 \mathrm{SCHZ}$ patients had an anomaly score larger than the healthy participants's mean and nine of them were larger than the 95th percentile of the healthy participants's population. In comparison, the difference between the anomaly scores was less pronounced for the PCA $(t=-1.75, P=0.08$, Cohen's $d=0.32)$ and $z$-score $(t=1.85, P=0.07$, Cohen's $d=0.33)$ approaches (see Supplementary Fig. 1 and Supplementary Table 2). Furthermore, the above chance-level detection rates of the proposed deep autoencoder in SCHZ suggest a successful application of the tractometry-based framework in unsupervised anomaly detection. The significance of these results are even more pronounced considering the challenging task at hand; that is, where even a supervised support vector machine classifier provides similar accuracy $(\mathrm{AUC}=0.65 \pm 0.13$ ). Finally, to provide an illustrative way in which the Detect tool can provide an important avenue for future studies, anomaly scores were correlated with clinical scores. The Hopkins anxiety index (Hopkins symptom checklist), a widely used screening instrument to study mental illness, was used as a proof of concept. For the autoencoder, PCA and $z$-score, the Spearman's rank correlation coefficients were $\rho=0.38(P=0.012), \rho=0.3(P=0.055)$ and $\rho=-0.12(P=0.448)$, respectively (Fig. 6).

Repeatability of anomaly scores and tract profiles. Using a testretest dataset (six patients, five time points ${ }^{29}$ ), we assessed the repeatability of (1) the input RISH0 tract profiles and (2) the generated anomaly scores by calculating the intraclass correlation coefficient (ICC, with two-way mixed, absolute agreement for average measurements as in ref. ${ }^{29}$ ) and the coefficient of variation $(\mathrm{CoV})$. Supplementary Fig. 2 shows the repeatability of the tract profiles, with the optic radiations being the most reproducible bundles (mean $\mathrm{ICC}=0.95, \mathrm{CoV}=0.03$ ) and the left cingulum being the least reproducible $(\mathrm{ICC}=0.66, \mathrm{CoV}=0.06)$. In terms of anomaly scores, the proposed anomaly detection framework shows reconstruction errors that are reproducible across sessions (Supplementary Fig. 3) with an ICC of 0.96 (95\% CI: 0.88, 0.99) and a CoV of 0.06. Furthermore, reliability of anomaly scores in the pediatric dataset can be estimated from the test-retest dataset. Using the two-way mixed ICC for consistency (for single measurements) of 0.85 from the test-retest study, assuming a similar measurement-related variance and accounting for the standard deviations in both cohorts, the ICC for anomaly scores in the pediatric cohort is 0.86 . Even if the measurement-related variance was to increase by $30 \%$, the ICC would still indicate good reliability $(0.76)$. This confirms that the demonstrated group differences in anomaly scores are unlikely to have occurred due to measurement-related variance.

\section{Discussion}

Further exploration of input features and hyperparameters of the model remains to assess the generalizability of the framework and its application to other pathologies. From a generalization point of view, the key problem in biomarker research is the need for individual prediction/diagnosis. Advancing knowledge of brain pathology and related cognitive impairment at the individual level is essential for early detection and intervention. Normative models show that, if groups are too heterogeneous, it can be a challenging task to learn characteristics from a given population using supervised approaches, hence the need for unsupervised learning ${ }^{1}$. Although the amount of data we can employ in imaging studies is relatively small in comparison with population-based studies, the framework provides a principled method to detect individual differences in tissue microstructure. With the ever-growing amount of dMRI data being acquired, the framework will make less conservative inferences as the number of data points increases. In principle, our framework can be trivially extended to tract-based assessment of non-diffusion-based microstructural metrics (for example, such as 


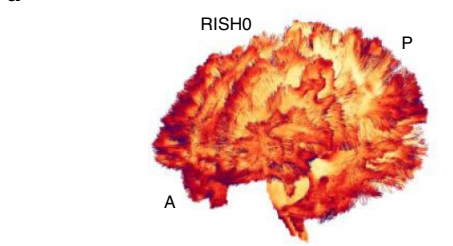

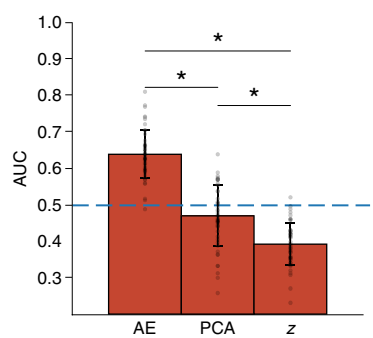
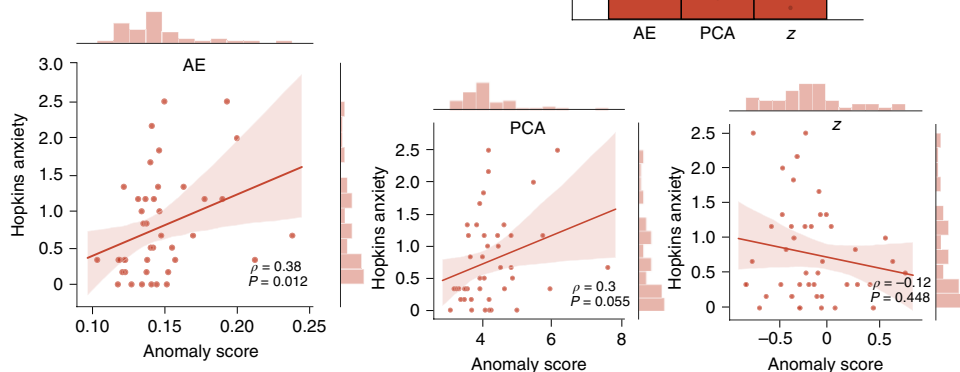

b

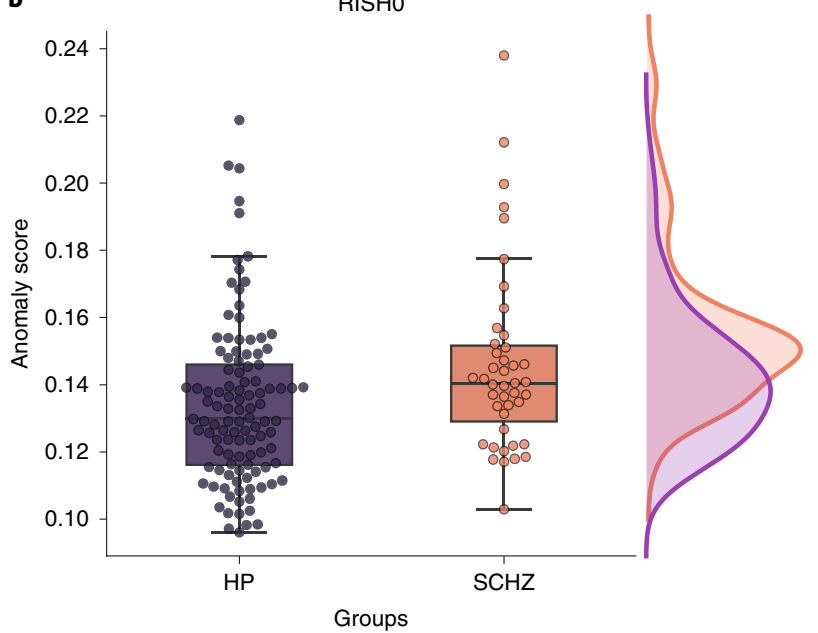

Fig. 6 | Anomaly scores for the SCHZ cohort. $\mathbf{a}$, The autoencoder provides a better discriminating power compared with traditional linear univariate and multivariate approaches with a mean AUC of $0.64 \pm 0.06$ (bar graph, ${ }^{\star} P=1.00 \times 10^{-33}, P=1.05 \times 10^{-5}$ and $P=1.63 \times 10^{-26}$, Bonferroni corrected at $\alpha=0.01$, two-tailed $t$-tests for AE-PCA, AE-z and PCA-z, respectively). For illustrative purposes, anomaly scores derived from the autoencoder, PCA and $z$-score are correlated with the Hopkins anxiety score (shaded area: 95\% confidence interval). b, The RISHO features show higher reconstruction error (100 averages) for the SCHZ than the healthy participants ( $t=-2.48, P=0.01$, Cohen's $d=0.47$, two-sided $t$-test; center line, median; box limits, upper and lower quartiles; whiskers, $1.5 \times$ interquartile range; $n=109$ healthy participants and $n=43$ SCZH, respectively).

magnetization transfer and myelin water imaging ${ }^{16,17}$ ), as long as the associated quantitative maps are co-registered with the diffusion space. They could be derived from any manually defined or atlasderived regions of interest, tract-based regions of interest as done here, or even at the voxel-based level ${ }^{39}$. Moreover, our framework could be applied to any set of neuroimaging features. For example, these inputs could include time series from functional MRI and cortical thickness derived from structural T1 imaging. Finally, other applications of the framework include the characterization of microstructural changes in neurological disorders without gross pathology. Furthermore, Detect could also help predict worse phenotypic outcome in those with a rare genetic disorder (for example, $\mathrm{CNV}$ carriers) and potentially allow for early therapeutic planning in the future.

One of the limitations of single-patient analysis is that it requires substantial amounts of healthy participant data to define a normative brain ${ }^{1}$. Combining multisite or multiscanner dMRI datasets can greatly increase the statistical power of neuroimaging studies ${ }^{40}$, but cross-scanner and cross-protocol variability challenges joint analysis, hence the need for data harmonization ${ }^{30,41}$. However, dMRI harmonization is a young field, there is not yet an established method that can bring cross-scanner variability back to the level of withinpatient variability. Moreover, tractography still faces considerable challenges in the field ${ }^{42,43}$ and most commonly available tools can only track reliably within normal-appearing white matter. Although recent machine learning approaches have shown promise in reproducible tract segmentation across patients ${ }^{44}$, therefore strengthening hope of analyzing dMRI data for group studies, there is a potential challenge: along-tract profiling approaches should only be used when a complete tract has been reconstructed. Future work will establish the utility of this approach in conditions where pathology leads to incomplete tract reconstruction. Another limitation is that autoencoders require more computation than PCA; however, PCA will also have limitations for large datasets where memory storage is an issue.

The choice of dMRI measure will also influence the capacity of the tool to detect anomalies. For example, the use of fiber-specific measurements ${ }^{45,46}$ could help better disentangle anomalies in fibercrossing regions. This would of course demand a model-based approach as opposed to the RISH0 features employed in this study. Furthermore, it was recently demonstrated that there is more sensitivity to individual differences at high $b$-values ${ }^{31,47}$. Nevertheless, we are encouraged to see that even with more commonly used $b$-values (for example, $b=1,000 \mathrm{~s} \mathrm{~mm}^{-2}$ ), we are still able to uncover patient/ control differences in the SCHZ cohort, highlighting the potential for widespread clinical adoption of dMRI.

In the context of microstructural MRI, the demonstration of Detect through the three different scenarios goes beyond the utility of other techniques and provides compelling motivation for future application. The unsupervised multivariate framework proposed here uses state-of-the-art machine learning to approach highdimensional data non-linearly and improve accuracy and precision over traditional anomaly detection. Our deep learning approach also provides advantages over other statistical approaches for outlier detection as it was recently shown (using functional MRI data ${ }^{3}$ ) that deep unsupervised approaches improve identification of psychiatric patients compared to mass-univariate normative modeling. It is also generally accepted that autoencoders tend to perform better when the middle layer (that is, bottleneck layer) is small when compared with PCA. This can potentially mean that the same accuracy can be achieved with less components and hence may be beneficial for smaller datasets.

Browser-based applications are becoming increasingly popular among the computational neuroscience community due to their ease of use and accessibility across devices ${ }^{21}$. Detect enables the detection of abnormalities in clinically heterogeneous groups or rare cases and ultimately improve diagnosis of neurological and neuropsychiatric disorders. Our aim was to develop and distribute an open-source framework to characterize microstructural whitematter changes at the individual level. This enables the detection of abnormalities To the best of our knowledge, other tools such as AFQ Browser ${ }^{21}$ compare individuals using a linear approach ( $z$-score) that considers each tract-segment independently and ignores potential complex interactions between the features. Those tract segments are then statistically tested in an univariate manner, and as such the correction for multiple comparisons-required by the typical high dimensionality of dMRI data-will hamper the discriminating power of the analysis ${ }^{25}$. Recently, PCA was employed 
to acknowledge the multivariate nature of dMRI data ${ }^{25-27}$, but this approach still relies on linear assumptions thereby ignoring possible complex interactions between the features. We believe strongly that the proposed deep autoencoder approach goes hand-in-hand with existing browser-based dMRI analysis frameworks ${ }^{21}$ in encouraging reproducible research and data-driven discoveries. Finally, we encourage future users of Detect to apply the tool to their own datasets and we welcome contribution to the tool in the form of added functionalities via GitHub.

In summary, diffusion MRI offers great promise to detect subtle differences in tissue microstructure when applied at the group level; however, the goal of clinical neuroimaging is to be applicable at the individual level. The single case approach proposed here will facilitate the identification of individualized therapy most appropriate to that patient, forming a baseline biomarker for subsequent monitoring through a therapeutic process. We believe that our tractometrybased anomaly detection framework paves the way to progress from the traditional paradigm of group-based comparison of patients against healthy participants, to a personalized medicine approach, and takes us a step closer in transitioning microstructural MRI from the bench to the beside.

\section{Methods}

Detect interactive interface. Users of Detect will input demographic data that consist of comma-separated values (.csv), where each row represents a patient (ID). Example demographics columns include: group, age, gender or clinical scores. The user is given the option to correct for confounding factors (that is, by treating those attributes as covariates across the entire brain ${ }^{2}$ ), resulting in age-independent microstructural features, for example. The microstructural tractometry data format consists of an .xlsx spreadsheet, where each sheet represents a dMRI metric (for example, fractional anisotropy, mean diffusivity and so on). As per the demographic data, patients are stacked individually on each row. The first column denotes the ID of each patient. The remaining columns follow the following convention: bundle_hemi_section where bundle is the white-matter bundle of interest, hemi is the hemisphere (that is, left or right and void for commissural tracts), and section is the along-tract portion (for example, from 1 to 20).

Data acquisition and preprocessing. $C N V$ dataset. Diffusion MRI data were acquired from 90 typically developing children (age 8-18 years) and eight children with CNVs at high risk of neurodevelopmental and psychiatric disorders (CNVs, $2 \times 15 q 13.3$ deletion, $2 \times 16$ p11.2 deletion, $3 \times 22 q .11 .2$ deletion and $1 \times$ Prader-Willi syndrome) and no apparent white-matter lesions (age $8-15$ years). Data collection procedures for the typically developing and CNV groups were approved by the Cardiff University School of Psychology and School of Medicine Ethics Committees, respectively. Children under 16 and those over 16 who lacked capacity to consent given written/verbal assent and their parents or legal guardians gave written consent on their behalf. Images were acquired using a Siemens $3 \mathrm{~T}$ Connectom MRI scanner (32-channel radiofrequency coil, Nova Medical) with $14 \mathrm{~b} 0$ images, 30 directions at $b=500,1,200 \mathrm{~s} \mathrm{~mm}^{-2} ; 60$ directions at $b=2,400$, $4,000,6,000 \mathrm{~s} \mathrm{~mm}^{-2}$; and $2 \times 2 \times 2 \mathrm{~mm}^{3}$ voxels (TE (echo time)/TR (repetition time $)=59 / 3,000 \mathrm{~ms} ; \Delta / \delta=24 / 7 \mathrm{~ms}$ ). The total scan time for the multishell protocol was $16 \mathrm{~min}$ and $14 \mathrm{~s}$. Each dataset was denoised ${ }^{48}$ and corrected for signal drift ${ }^{49}$, motion and distortion in $\mathrm{FSL}^{50}$, gradient non-linearities ${ }^{51}$ and Gibbs ringing ${ }^{52}$ Next, RISH features ${ }^{30}$ were derived for each patient using the $b=6,000 \mathrm{~s} \mathrm{~mm}^{-2}$ shell to maximize sensitivity to the intra-axonal signal ${ }^{31}$ (zeroth and second orders only, RISH0 and RISH2, respectively). Furthermore, diffusion tensors were generated using an in-house non-linear least squares fitting routine using only the $b \leq 1,200 \mathrm{~s} \mathrm{~mm}^{-2}$ data, followed by the derivation of fractional anisotropy and mean diffusivity maps.

Epilepsy dataset. Diffusion MRI data from two epilepsy patients with FCD were acquired on a Siemens $3 \mathrm{~T}$ Connectom MRI scanner with 60 directions at $b=1,200$, 3,000 and $5,000 \mathrm{~s} \mathrm{~mm}^{-2}$ and $1.2 \times 1.2 \times 1.2 \mathrm{~mm}^{3}$ voxels $(\mathrm{TE} / \mathrm{TR}=68 / 5,400 \mathrm{~ms}$; $\Delta / \delta=31.1 / 8.5 \mathrm{~ms}$; total scan time $=30 \mathrm{~min}$ and $25 \mathrm{~s}$ ). Furthermore, data on 15 healthy participants (aged 21-41 years) from the computational diffusion MRI harmonization database were used ${ }^{40}$. Data collection procedures for the healthy participant and FCD groups were approved by the Cardiff University School of Psychology and School of Medicine Ethics Committees, respectively. Written informed consent was obtained from all patients. Each dataset was corrected for Gibbs ringing ${ }^{52}$, signal drift ${ }^{49}$, motion and distortion in $\mathrm{FSL}^{50}$, and gradient non-linearities ${ }^{51}$. Next, RISH0 features ${ }^{30}$ were derived for each patient using the $b=5,000 \mathrm{~s} \mathrm{~mm}^{-2}$ shell. A fourfold data augmentation was applied to the healthy participant tract profiles using the synthetic minority oversampling technique $\mathrm{e}^{53}$ resulting in 75 healthy participants.
SCHZ dataset. Diffusion MRI data from the UCLA Consortium for Neuropsychiatric Phenomics ${ }^{54}$ was downloaded from the OpenNeuro platform (openneuro.org/datasets/ds000030/versions/00016), which also contains demographic, behavioral and clinical data. Although more focused on functional MRI, the dataset contains dMRI data from 123 healthy participants and 49 individuals with SCHZ amongst other psychiatric disorders. Data were acquired on a Siemens $3 \mathrm{~T}$ Tim Trio MRI scanner with one b0 image, 64 directions at $b=1,000 \mathrm{~s} \mathrm{~mm}^{-2}$ and $2 \times 2 \times 2 \mathrm{~mm}^{3}$ voxels. Data quality assessment was first performed, resulting in the exclusion of datasets with reduced field-of-view (preventing the reconstruction of white-matter bundles in the inferior temporal lobes) and those with substantial slice dropout (impacting estimation of diffusion metrics). A total number of 109 healthy participants (aged 21-50 years) and $43 \mathrm{SCHZ}$ patients (aged 22-49 years) were used for further analysis. Diffusion data were denoised ${ }^{48}$, corrected for patient motion in $\mathrm{FSL}^{50}$ and distortion using the anatomical T1-weighted image as reference. Next, RISH features (RISH0, RISH2) were derived for each patient. Finally, diffusion tensors were generated using iteratively weighted least squares in $\mathrm{MRtrix}^{55}$ followed by the derivation of fractional anisotropy and mean diffusivity maps.

Repeatability dataset. To assess repeatability, we employed the microstructural image compilation with repeated acquisitions dataset ${ }^{29}$, which comprises five repeated sets of microstructural imaging in six healthy human participants (three female, aged 24-30 years). Each participant was scanned five times in the span of two weeks on a $3 \mathrm{~T}$ Siemens Connectom system with ultra-strong $\left(300 \mathrm{mT} \mathrm{m}^{-1}\right)$ gradients. Multishell dMRI data were collected $(\mathrm{TE} / \mathrm{TR}=59 / 3,000 \mathrm{~ms}$; voxel size $=2 \times 2 \times 2 \mathrm{~mm}^{3} ; b$-values $=0$ (14 volumes), 200 and 500 (20 directions), 1,200 (30 directions), 2,400,4,000, and 6,000 (60 directions) $\mathrm{s} \mathrm{mm}^{-2}$ ) resulting in a total scan time of $16 \mathrm{~min}$ and $37 \mathrm{~s}$. The same preprocessing as the CNV dataset was applied. Data collection was approved by the Cardiff University School of Psychology Ethic Committee and written informed consent was obtained from all patients.

Tractometry. For each dataset, automated white-matter tract segmentation was performed using TractSeg ${ }^{44}$ (see Supplementary Table 1) using multishell constrained spherical deconvolution ${ }^{47}$. For each bundle, 2,000 streamlines were generated. Tractometry ${ }^{16}$ was performed (sampling fractional anisotropy, mean diffusivity, and RISH0 and RISH2 at 20 locations along the tracts ${ }^{19,23,25}$ ) using a Nextflow architecture provided by SCILPY (github.com/scilus/scilpy). Specifically, individual streamlines were reordered for all patients to ensure consistency using the following order: left-to-right for commissural tracts, anterior-to-posterior for association pathways and top-to-bottom for projection pathways. Next, a core streamline was generated and microstructural metrics at each vertex of the bundle were projected to the closest point along the core. The resulting tract profiles were concatenated to form a feature vector $(x)$

Artificial neural network. Our autoencoder implementation consists of a symmetric design of five fully connected layers $\left(1_{n}\right)$. The input and output layers $\left(l_{1}\right.$ and $\left.l_{5}\right)$ have exactly the same number of nodes as the number of input tracts features. The inner layers $\left(\mathrm{l}_{2}\right.$ and $\left.\mathrm{l}_{4}\right)$ consecutively apply a compression ratio of two by reducing the number of nodes by half, up to the bottleneck hidden layer $\left(l_{3}\right)$. For example, if using an input vector made of 100 features, $l_{1}$ and $l_{5}$ will consist of 100 nodes; $l_{2}$ and $l_{4} 50$ nodes; and 25 nodes for the bottleneck $l_{3}$. Rectified linear units activation was used between the layers to promote sparse activation and tan $h$ for the last layer (epochs $=25$, batch size $=24$, learning rate $=1 \times 10^{-3}$; optimizer, Adam; loss, mean squared error; validation split $=0.1$ ). Using different activation functions in different layers aims at balancing the advantages and disadvantages of the two activation functions. To promote sparsity and reduce overfitting, an activation penalty was imposed to the bottleneck layer using $\ell_{1}$-regularization $\left(\times 10^{-5}\right)$. This is especially best suited for models that explicitly seek an efficient learned representation. The goal is to generate an output $(\hat{x})$ similar to the input $(x)$ by minimizing the reconstruction error. Here the MAE was used as anomaly score and is defined as:

$$
\mathrm{MAE}=\frac{1}{n} \sum_{j=1}^{n}\left|x_{i}-\hat{x}_{i}\right|,
$$

The MAE measures the average magnitude of the errors and is derived during testing by computing the absolute differences between the reconstructed microstructural features $\left(\hat{x}_{i}\right)$ and the raw input features $\left(x_{i}\right)$. Due to the heavily imbalanced group ratio between healthy participants and patients (that is, CNV and epilepsy), a bootstrap was implemented to draw random samples of equal sizes from each group. Specifically, the autoencoder is trained using healthy participants data only. The entire dataset is therefore first split into a training set $(80 \%$, made of healthy controls only) and a validation set ( $20 \%$, by combining the patients with a matching number of healthy participants) to create the outer fold; $10 \%$ of the training set is held out for testing during the training phase (inner fold) to evaluate the loss. Age and sex regression ${ }^{56}$ and feature normalization (min-max) were performed on the normative training set and subsequently applied to the held-out validation set to prevent information leakage. To derive conservative estimates 
and assess variations within the model, we repeated this process 100 times and report the mean MAE for each patient. Finally, we compared the sensitivity versus specificity of the anomaly scores using the mean receiver operating characteristic AUC across iterations, with standard deviations used as uncertainties. Comparisons of the AUC scores between patients and controls were performed using two-tailed $t$-tests assuming equal variances in the case of balanced groups and Kolmogorov-Smirnov test for the unbalanced groups. The correlation between anomaly scores and clinical scores was computed using Spearman's $\rho$.

Univariate approach. One of the most commonly used tools in determining outliers is the $z$-score. The $z$-score (or standard score $z=x-\mu$ ) $/ \sigma$ ) is a way of describing a data point as deviance from distribution, in terms of standard deviations from the mean of the normal distribution. Here, $z$-scores were computed for each tract-segment, relative to the mean of the healthy group $(\mu)$ and averaged to derive a patient-specific anomaly score at each iteration (described above). The mean over all iterations described above was retained as anomaly scores for all patients.

Multivariate linear approach. Principal component analysis was applied to the set of features by restricting the dimensionality to preserve features accounting for $85 \%$ of the variance in the data. In Detect, this number can be manually defined as the percentage of explained variance. Next, the Mahalanobis Distance $(M$, a multidimensional generalization of the $\mathrm{z}$-score that accounts for the relationships between the white-matter bundles) was used to derive an anomaly score defined as:

$$
M(x)=\sqrt{(x-\mu)^{\prime} \cdot C^{-1} \cdot(x-\mu)},
$$

where $x$ represents the feature vector of a given patient, $\mu$ is the vector of mean microstructural metrics for each tract location $s$, and $C^{-1}$ is the inverse covariance matrix of the input features. The problem of anomaly detection can be seen as a one-class classification problem and therefore, our training data only contains healthy participants to calculate $C$. an $M$ score was then derived for all unseen patients in relation to the healthy participant distribution. The same dataset split as aforementioned was used to derive a bootstrapped estimate of $M$ for each patient, which was subsequently analyzed.

Support vector machine comparison. A supervised support vector machine classifier was used for comparisons on the SCHZ dataset. Class weights were set to account for the class imbalance between healthy participants and patients. The classifier was validated using a repeated (ten times) stratified, fivefold crossvalidation approach in scikit-learn (scikit-learn.org). Optimized parameters were derived for each cross-validation fold using a grid-search approach. Those included the choice of kernel ([radial basis function, linear]), regularization $([1,10,100$, $1000])$ and gamma parameters $\left(\left[10^{-3}, 10^{-2}, 10^{-1}, 1,10^{1}, 10^{2}, 10^{3}\right]\right)$

Reporting Summary. Further information on research design is available in the Nature Research Reporting Summary linked to this article.

\section{Data availability}

Original datasets are accessible through the original publications, including the MICRA ${ }^{29}$ repeatability dataset (osf.io/z3mkn/) and the $\mathrm{CNP}^{54}$ dataset (https:// openneuro.org/datasets/ds000030/versions/1.0.00). The cDMRI dataset is publicly available and information on how to obtain the data can be found on the following webpage: https://forms.office.com/r/ZyLNjuYk3Y. Source Data are provided with this paper.

\section{Code availability}

Detect is an open-source anomaly detection framework for neuroimaging data and is available through Github ${ }^{57}$ at github.com/chamberm/Detect under the Apache License. The framework is powered by Streamlit (www.streamlit.io), an open-source app framework for machine learning and data science. The repository is regularly updated via continuous integration to contain example data (tractprofiles), a Wiki section as well as Jupyter Notebooks with the Python code used to generate the figures in this study. A live demo is also available via Streamlit sharing. TractSeg is available at github.com/MIC-DKFZ/TractSeg. MRtrix is available at www.mrtrix.org. SCILPY is available at github.com/scilus/scilpy. FiberNavigator is available at github.com/chamberm/fibernavigator.

Received: 18 February 2021; Accepted: 9 August 2021; Published online: 22 September 2021

\section{References}

1. Marquand, A. F., Rezek, I., Buitelaar, J. \& Beckmann, C. F. Understanding heterogeneity in clinical cohorts using normative models: beyond casecontrol studies. Biol. Psychiatry 80, 552-561 (2016).

2. Marquand, A. F. et al. Conceptualizing mental disorders as deviations from normative functioning. Mol. Psychiatry 24, 1415-1424 (2019).
3. Kia, S. \& Marquand, A. Neural processes mixed-effect models for deep normative modeling of clinical neuroimaging data. In Proceedings of The 2nd International Conference on Medical Imaging with Deep Learning (eds. Cardoso, M. J. et al.) Vol. 102, 297-314 (PMLR, 2019).

4. Wolfers, T. et al. Mapping the heterogeneous phenotype of schizophrenia and bipolar disorder using normative models. JAMA Psychiatry 75, 1146-1155 (2018).

5. Pinaya, W. H. L., Mechelli, A. \& Sato, J. R. Using deep autoencoders to identify abnormal brain structural patterns in neuropsychiatric disorders: a large-scale multi-sample study. Hum. Brain Mapp. 40, 944-954 (2019).

6. Stejskal, E. O. \& Tanner, J. E. Spin diffusion measurements: spin echoes in the presence of a time-dependent field gradient. J. Chem. Phys. 42, 288-292 (1965).

7. Pierpaoli, C. \& Basser, P. J. Toward a quantitative assessment of diffusion anisotropy. Magn. Reson. Med. 36, 893-906 (1996).

8. Basser, P. J., Mattiello, J. \& LeBihan, D. Estimation of the effective selfdiffusion tensor from the NMR spin echo. J. Magn. Reson. B 103, 247-254 (1994).

9. Conturo, T. E. et al. Tracking neuronal fiber pathways in the living human brain. Proc. Natl Acad. Sci. USA 96, 10422-10427 (1999).

10. Jones, D. K. \& Cercignani, M. Twenty-five pitfalls in the analysis of diffusion MRI data. NMR Biomed. 23, 803-820 (2010).

11. Hong, S.-J. et al. Towards neurosubtypes in autism. Biol. Psychiatry 88, 111-128 (2020)

12. Deleo, F. et al. Histological and mri markers of white matter damage in focal epilepsy. Epilepsy Res. 140, 29-38 (2018).

13. Scholz, J., Tomassini, V. \& Johansen-Berg, H. Individual differences in white matter microstructure in the healthy brain. In Diffusion MRI (Second Edition) (eds. Johansen-Berg, H. \& Behrens, T. E.) Ch. 14, 301-316 (Academic, 2014); https://doi.org/10.1016/B978-0-12-396460-1.00014-7

14. Lv, J. et al. Individual deviations from normative models of brain structure in a large cross-sectional schizophrenia cohort. Mol. Psychiatry https://doi. org/10.1038/s41380-020-00882-5 (2020).

15. Jones, D. K. et al. Age effects on diffusion tensor magnetic resonance imaging tractography measures of frontal cortex connections in schizophrenia. Hum. Brain Mapp. 27, 230-238 (2006).

16. Bells, S. et al. Tractometry-comprehensive multi-modal quantitative assessment of white matter along specific tracts. In Proc. ISMRM Vol. 678, 0678 (2011).

17. De Santis, S., Drakesmith, M., Bells, S., Assaf, Y. \& Jones, D. K. Why diffusion tensor MRI does well only some of the time: variance and covariance of white matter tissue microstructure attributes in the living human brain. NeuroImage 89, 35-44 (2014).

18. Jones, D. K., Travis, A. R., Eden, G., Pierpaoli, C. \& Basser, P. J. PASTA: pointwise assessment of streamline tractography attributes. Magn. Reson. Med. 53, 1462-1467 (2005).

19. Yeatman, J. D., Dougherty, R. F., Myall, N. J., Wandell, B. A. \& Feldman, H. M. Tract profiles of white matter properties: automating fiber-tract quantification. PLoS ONE 7, e49790 (2012).

20. Colby, J. B. et al. Along-tract statistics allow for enhanced tractography analysis. NeuroImage 59, 3227-3242 (2012).

21. Yeatman, J. D., Richie-Halford, A., Smith, J. K., Keshavan, A. \& Rokem, A. A browser-based tool for visualization and analysis of diffusion MRI data. Nat. Commun. 9, 940 (2018).

22. Dayan, M. et al. Optic radiation structure and anatomy in the normally developing brain determined using diffusion MRI and tractography. Brain Struct. Funct. 220, 291-306 (2015).

23. Cousineau, M. et al. A test-retest study on Parkinson's PPMI dataset yields statistically significant white matter fascicles. NeuroImage Clin.16, 222-233 (2017).

24. Sarica, A. et al. The corticospinal tract profile in amyotrophic lateral sclerosis. Hum. Brain Mapp. 38, 727-739 (2017).

25. Chamberland, M. et al. Dimensionality reduction of diffusion MRI measures for improved tractometry of the human brain. NeuroImage 200, 89-100 (2019).

26. Dean III, D. et al. Multivariate characterization of white matter heterogeneity in autism spectrum disorder. NeuroImage Clin. 14, 54-66 (2017).

27. Taylor, P. N., Moreira da Silva, N., Blamire, A., Wang, Y. \& Forsyth, R. Early deviation from normal structural connectivity: a novel intrinsic severity score for mild TBI. Neurology 94, e1021-e1026 (2020).

28. Hinton, G. E. \& Salakhutdinov, R. R. Reducing the dimensionality of data with neural networks. Science 313, 504-507 (2006).

29. Koller, K. et al. MICRA: Microstructural image compilation with repeated acquisitions. Neuroimage 225, 117406 (2021).

30. Mirzaalian, $H$. et al. Inter-site and inter-scanner diffusion mri data harmonization. NeuroImage 135, 311-323 (2016).

31. Genc, S. et al. Impact of b-value on estimates of apparent fibre density. Hum. Brain Mapp. 41, 2583-2595 (2020).

32. Chawner, S. J. et al. Genotype-phenotype associations in children with copy number variants associated with high neuropsychiatric risk in the UK (imagine-id): a case-control cohort study. Lancet Psych. 6, 493-505 (2019). 
33. Villalón-Reina, J. E. et al. Altered white matter microstructure in $22 \mathrm{q} 11.2$ deletion syndrome: a multisite diffusion tensor imaging study. Mol Psychiatry 25, 2818-2831 (2020).

34. Tylee, D. S. et al. Machine-learning classification of 22q11.2 deletion syndrome: a diffusion tensor imaging study. NeuroImage Clin. $\mathbf{1 5}$, 832-842 (2017).

35. Lerner, J. T. et al. Assessment and surgical outcomes for mild type I and severe type II cortical dysplasia: a critical review and the UCLA experience. Epilepsia 50, 1310-1335 (2009).

36. Duncan, J. S., Winston, G. P., Koepp, M. J. \& Ourselin, S. Brain imaging in the assessment for epilepsy surgery. Lancet Neurol. 15 420-433 (2016).

37. Blümcke, I. et al. The clinicopathologic spectrum of focal cortical dysplasias: a consensus classification proposed by an ad hoc Task Force of the ILAE Diagnostic Methods Commission. Epilepsia 52, 158-174 (2011).

38. Stephan, K. E., Friston, K. J. \& Frith, C. D. Dysconnection in schizophrenia: from abnormal synaptic plasticity to failures of self-monitoring. Schizophrenia Bull. 35, 509-527 (2009).

39. Smith, S. M. et al. Tract-based spatial statistics: voxelwise analysis of multi-subject diffusion data. NeuroImage 31, 1487-1505 (2006).

40. Tax, C. M. et al. Cross-scanner and cross-protocol diffusion MRI data harmonisation: a benchmark database and evaluation of algorithms. NeuroImage 195, 285-299 (2019).

41. Cetin Karayumak, S. et al. Retrospective harmonization of multi-site diffusion MRI data acquired with different acquisition parameters. NeuroImage $\mathbf{1 8 4}$ 180-200 (2019).

42. Maier-Hein, K. H. et al. The challenge of mapping the human connectome based on diffusion tractography. Nat. Commun. 8, 1349 (2017).

43. Jones, D. K., Knösche, T. R. \& Turner, R. White matter integrity, fiber count, and other fallacies: the do's and don'ts of diffusion MRI. NeuroImage 73, 239-254 (2013).

44. Wasserthal, J., Neher, P. \& Maier-Hein, K. H. TractSeg_fast and accurate white matter tract segmentation. NeuroImage 183, 239-253 (2018).

45. Raffelt, D. et al. Apparent fibre density: a novel measure for the analysis of diffusion-weighted magnetic resonance images. NeuroImage 59, 3976-3994 (2012)

46. Assaf, Y. \& Basser, P. J. Composite hindered and restricted model of diffusion (CHARMED) MR imaging of the human brain. NeuroImage 27, 48-58 (2005)

47. Jeurissen, B., Tournier, J.-D., Dhollander, T., Connelly, A. \& Sijbers, J. Multi-tissue constrained spherical deconvolution for improved analysis of multi-shell diffusion MRI data. NeuroImage 103, 411-426 (2014).

48. Veraart, J. et al. Denoising of diffusion MRI using random matrix theory. NeuroImage 142, 394-406 (2016).

49. Vos, S. B. et al. The importance of correcting for signal drift in diffusion MRI. Magn. Reson. Med. 77, 285-299 (2017).

50. Jenkinson, M., Beckmann, C. F., Behrens, T. E., Woolrich, M. W. \& Smith, S. M. FSL. NeuroImage 62, 782-790 (2012).

51. Glasser, M. F. et al. The minimal preprocessing pipelines for the Human Connectome Project. NeuroImage 80, 105-124 (2013).

52. Kellner, E., Dhital, B., Kiselev, V. G. \& Reisert, M. Gibbs-ringing artifact removal based on local subvoxel-shifts. Magn. Reson. Med. 76, 1574-1581 (2016).

53. Chawla, N. V., Bowyer, K. W., Hall, L. O. \& Kegelmeyer, W. P. SMOTE: synthetic minority over-sampling technique. J. Artif. Intelli. Res.16, 321-357 (2002).

54. Poldrack, R. A. et al. A phenome-wide examination of neural and cognitive function. Sci. Data 3, 1-12 (2016).

55. Tournier, J.-D. et al. MRtrix3: a fast, flexible and open software frame work for medical image processing and visualisation. NeuroImage 202, 116137 (2019)
56. Lebel, C. et al. Diffusion tensor imaging of white matter tract evolution over the lifespan. NeuroImage 60, 340-352 (2012).

57. Chamberland, M. chamberm/Detect: Detect (Zenodo, 2021); https://doi. org/10.5281/zenodo.4945138

\section{Acknowledgements}

This work was supported by a Wellcome Trust Investigator Award (grant no. 096646/Z/11/Z), a Wellcome Trust Strategic Award (grant no. 104943/Z/14/Z), and an EPSRC equipment grant (no. EP/M029778/1) to D.K.J., a Sir Henry Wellcome Fellowship (grant no. 215944/Z/19/Z) and VENI grant (no. 17331) from the Dutch Research Council (NWO) to C.M.W.T., a Wellcome Trust GW4-CAT Fellowship (grant no. 220537/Z/20/Z) to D.S., a Wellcome Trust Fellowship (grant no. 102003/Z/13/Z) to J.D. and an NIH NICDH fellowship (grant no. 1F32HD103313-01) to EPR. This study is also supported by the Brain Repair and Intracranial Neurotherapeutics (BRAIN) Unit, funded by Welsh Government through Health and Care Research Wales. M.C. was in part supported by the Radboud Excellence Initiative Fellowship. The authors thank M. Descoteaux and J.-C. Houde (Sherbrooke Connectivity Imaging Laboratory) for their useful discussions and code sharing.

\section{Author contributions}

M.C. developed the framework, conducted the experiments, analyzed the results, and wrote the manuscript, M.C., C.M.W.T. and D.K.J. conceptualized the project, A.C., J.D. and M.v.d.B. recruited the $\mathrm{CNV}$ patients, S.G. and E.P.R. acquired the pediatric data, M.C. and S.G. pre-processed the pediatric imaging data, G.D.P. and C.M.W.T. developed the preprocessing pipeline, W.P.G., D.S. and K.H procured the epilepsy data, M.C., C.M.W.T. and D.S. acquired and pre-processed the cdMRI and epilepsy data, K.K. acquired the MICRA repeatability data. All authors reviewed and contributed to the manuscript.

\section{Competing interests}

The authors declare no competing interests.

\section{Additional information}

Supplementary information The online version contains supplementary material available at https://doi.org/10.1038/s43588-021-00126-8.

Correspondence and requests for materials should be addressed to Maxime Chamberland.

Peer review information Nature Computational Science thanks Laurent Petit, Daniel C. Alexander and the other, anonymous, reviewer(s) for their contribution to the peer review of this work.

Editor recognition statement Handling editor: Ananya Rastogi, in collaboration with the Nature Computational Science team.

Reprints and permissions information is available at www.nature.com/reprints.

Publisher's note Springer Nature remains neutral with regard to jurisdictional claims in published maps and institutional affiliations.

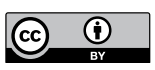

Open Access This article is licensed under a Creative Commons Attribution 4.0 International License, which permits use, sharing, adaptation, distribution and reproduction in any medium or format, as long as you give appropriate credit to the original author(s) and the source, provide a link to the Creative Commons license, and indicate if changes were made. The images or other third party material in this article are included in the article's Creative Commons license, unless indicated otherwise in a credit line to the material. If material is not included in the article's Creative Commons license and your intended use is not permitted by statutory regulation or exceeds the permitted use, you will need to obtain permission directly from the copyright holder. To view a copy of this license, visit http://creativecommons.org/licenses/by/4.0/.

(c) The Author(s) 2021 


\section{Reporting Summary}

Nature Research wishes to improve the reproducibility of the work that we publish. This form provides structure for consistency and transparency in reporting. For further information on Nature Research policies, see our Editorial Policies and the Editorial Policy Checklist.

\section{Statistics}

For all statistical analyses, confirm that the following items are present in the figure legend, table legend, main text, or Methods section.

$\mathrm{n} / \mathrm{a}$ Confirmed

$\bigotimes$ The exact sample size $(n)$ for each experimental group/condition, given as a discrete number and unit of measurement

\ A statement on whether measurements were taken from distinct samples or whether the same sample was measured repeatedly

The statistical test(s) used AND whether they are one- or two-sided

Only common tests should be described solely by name; describe more complex techniques in the Methods section.

$\bigotimes$ A description of all covariates tested

Х A description of any assumptions or corrections, such as tests of normality and adjustment for multiple comparisons

A full description of the statistical parameters including central tendency (e.g. means) or other basic estimates (e.g. regression coefficient)

AND variation (e.g. standard deviation) or associated estimates of uncertainty (e.g. confidence intervals)

For null hypothesis testing, the test statistic (e.g. $F, t, r$ ) with confidence intervals, effect sizes, degrees of freedom and $P$ value noted Give $P$ values as exact values whenever suitable.

Х $\square$ For Bayesian analysis, information on the choice of priors and Markov chain Monte Carlo settings

Х $\square$ For hierarchical and complex designs, identification of the appropriate level for tests and full reporting of outcomes

$\square \bigotimes$ Estimates of effect sizes (e.g. Cohen's $d$, Pearson's $r$ ), indicating how they were calculated

\section{Our web collection on statistics for biologists contains articles on many of the points above.}

\section{Software and code}

Policy information about availability of computer code

Data collection Data was acquired on a 3.0T Siemens Connectom system with ultra-strong $(300 \mathrm{mT} / \mathrm{m})$ gradients.

Data analysis Detect is available through Github at github.com/chamberm/Detect and https://doi.org/10.5281/zenodo.4945138.

The framework is powered by Streamlit (v0.53) www.streamlit.io, an open-source app framework for machine learning and data science.

The code used to generate some of the figures is available at github.com/chamberm/Detect/tree/master/examples

TractSeg (v2.2) is available at github.com/MIC-DKFZ/TractSeg.

MRtrix (3.0.2) is available at www.mrtrix.org.

FSL (5.0.9) is available at fsl.fmrib.ox.ac.uk/fsl/fslwiki

SCILPY (1.0.0) is available at github.com/scilus/scilpy.

FiberNavigator (Fall 2019) is available at github.com/chamberm/fibernavigator.

For manuscripts utilizing custom algorithms or software that are central to the research but not yet described in published literature, software must be made available to editors and

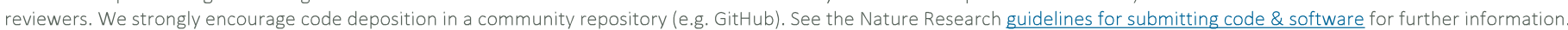


Policy information about availability of data

All manuscripts must include a data availability statement. This statement should provide the following information, where applicable:

- Accession codes, unique identifiers, or web links for publicly available datasets

- A list of figures that have associated raw data

- A description of any restrictions on data availability

The MICRA repeatability dataset is available at osf.io/z3mkn.

Diffusion MRI data from the UCLA Consortium for Neuropsychiatric Phenomics (Poldrack et al. 2016) was downloaded from the OpenNeuro platform (openneuro.org/datasets/ds000030/versions/00016).

The cdMRI dataset is available at: https://www.cardiff.ac.uk/cardiff-university-brain-research-imaging-centre/research/projects/cross-scanner-and-cross-protocoldiffusion-MRI-data-harmonisation.

Tract-profile data are available via the github repository (github.com/chamberm/Detect) and at https://doi.org/10.5281/zenodo.4945138.

\section{Field-specific reporting}

Please select the one below that is the best fit for your research. If you are not sure, read the appropriate sections before making your selection.

$\bigotimes$ Life sciences $\quad \square$ Behavioural \& social sciences $\quad \square$ Ecological, evolutionary \& environmental sciences

For a reference copy of the document with all sections, see nature.com/documents/nr-reporting-summary-flat.pdf

\section{Life sciences study design}

All studies must disclose on these points even when the disclosure is negative.

Sample size The analysis pipeline presented in this paper relies on a normative approach. Below are the sample sizes for each dataset:

CNV dataset: 90 typically developing (TD) children +8 children with copy-number variants (CNV) at high risk of neurodevelopmental and psychiatric disorders.

CNP dataset: 109 healthy controls $(\mathrm{HC})+43$ individuals with schizophrenia (SCHZ)

cdMRI dataset: 15 healthy controls $(\mathrm{HC})+2$ epilepsy patients with focal cortical dysplasia (FCD). A four-fold data augmentation was applied to the HC tract-profiles using a Synthetic Minority Over-sampling TEchnique (SMOTE)

MICRA repeatability data: 6 healthy controls were scanned 5 times each. Power calculation can be found in the original publication: Koller et al. 2020 Neuroimage.

Data exclusions CNP dataset: Data quality assessment was first performed, resulting in the exclusion of datasets with reduced field-of-view (preventing the reconstruction of white matter bundles in the inferior temporal lobes) and those with significant slice dropout (impacting estimation of diffusion metrics).

Replication To assess repeatability, we employed the microstructural image compilation with repeated acquisitions dataset (MICRA, Koller et al. 2020), which comprises 5 repeated sets of microstructural imaging in 6 healthy human participants ( 3 female, age 24-30 years). Each participant was scanned five times in the span of two weeks on a 3.0T Siemens Connectom system with ultra-strong ( $300 \mathrm{mT} / \mathrm{m})$ gradients. Within-subject variance was lower than between subject variance, which indicates that group differences in anomaly scores are unlikely to have occurred due to measurement-related variance.

Randomization This is not relevant to this study since all samples and subjects are in the same group.

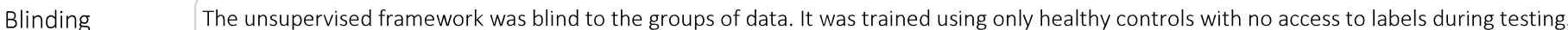

\section{Reporting for specific materials, systems and methods}

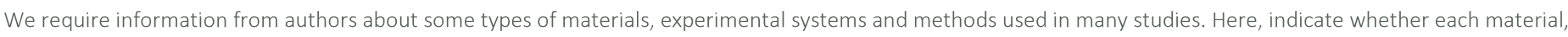

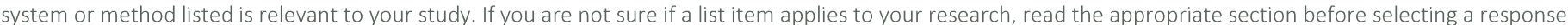




\begin{tabular}{l|l}
\hline n/a & Involved in the study \\
$\square$ & $\square$ Antibodies \\
$\square$ & $\square$ Eukaryotic cell lines \\
$\square$ & $\square$ Palaeontology and archaeology \\
$\square$ & $\square$ Animals and other organisms \\
$\square$ & $\square$ Human research participants \\
$\square$ & $\square$ Clinical data \\
$\square$ & $\square$ Dual use research of concern
\end{tabular}

\section{Human research participants}

\section{Policy information about studies involving human research participants}

Population characteristics

CNV dataset: Diffusion MRI data were acquired from 90 typically developing (TD, 47 female, age 8-18 years) and 8 children with copy-number variants at high risk of neurodevelopmental and psychiatric disorders (CNVs) and no apparent WM lesions (4 female, age 8-15 years).

cdMRI dataset: Diffusion MRI data from 2 epilepsy patients with focal cortical dysplasia (FCD, 2 female, age 20-47 years) were acquired on a Siemens 3T Connectom MRI scanner. In addition, 15 healthy controls (HC) (11 female, age 21-41 years) from the computational diffusion MRI harmonization database were used (Tax et al. 2019).

MICRA dataset: To assess repeatability, we employed the microstructural image compilation with repeated acquisitions dataset (MICRA, Koller et al. 2020), which comprises 5 repeated sets of microstructural imaging in 6 healthy human participants ( 3 female, age 24-30 years). Each participant was scanned five times in the span of two weeks on a 3.0T Siemens Connectom system with ultra-strong $(300 \mathrm{mT} / \mathrm{m})$ gradients.

CNP dataset: 109 healthy controls (55 female, age 21-50 years) and 43 individuals with schizophrenia (SCHZ, 9 female, age 22-49 years)

Recruitment

Healthy subjects (MICRA, cdMRI) are employees, students and friends of staff at Cardiff University Brain Research Imaging Centre.

Typically developing children were recruited from the Cardiff area, and screened to exclude major neurological disorders, such as epilepsy. CNV participants were recruited from the Experiences of CHildren with cOpy (ECHO) number variants study at Cardiff University (https://bit.ly/Cardiff-Echo-Study).

Epilepsy subjects were recruited from Cardiff \& Vale University Health Board Epilepsy Surgery program that receives referrals from Neurology departments across South Wales. All patients going through the program are invited to participate in research and their neuroimaging data are acquired ahead of the invasive procedures. The research undertaken does not trial diagnostic or therapeutic interventions and does not affect clinical management of the patients in any way.

Ethics oversight

Data collection procedures for the TD and CNV groups were approved by the Cardiff University School of Psychology and School of Medicine Ethics Committees, respectively. Written informed consent was obtained from all participants and legal guardians.

Data collection procedures for the HC and FCD groups were approved by the Cardiff University School of Psychology and School of Medicine Ethics Committees, respectively. Written informed consent was obtained from all subjects.

MICRA: Data collection was approved by the Cardiff University School of Psychology Ethic Committee and written informed consent was obtained from all subjects.

Note that full information on the approval of the study protocol must also be provided in the manuscript.

\section{Magnetic resonance imaging}

\section{Experimental design}

Design type

\section{Design specifications}

Behavioral performance measures
Diffusion MRI, brain imaging

Scan time of 20 minutes

CNV, FCD, CDMRI and MICRA dataset: No behavioral measures were investigated in this study. SCZH: Hopkins Anxiety Score from the CNP Dataset available online at: (openneuro.org/datasets/ds000030/ versions/00016). 
Acquisition

Imaging type(s)

Field strength

Sequence \& imaging parameters

Area of acquisition

Diffusion MRI

Parameters

Used

Not used

\section{Whole brain}

CNV: Images were acquired using a Siemens $3 T$ Connectom MRI scanner with ultra-strong ( $300 \mathrm{mT} / \mathrm{m}$ ) gradients using single-shot spin echo, echo planar imaging (TE/TR $=59 / 3000 \mathrm{~ms}, 1.2 \times 1.2 \times 1.2 \mathrm{~mm}^{3}$ voxels). The field of view was $220 \times$ $220 \mathrm{~mm}$ in plane, the matrix size was $110 \times 110 \times 66$. Total scan time: 16 minutes and 14 seconds.

cdMRI: Images were acquired on a Siemens 3T Connectom MRI scanner with ultra-strong ( $300 \mathrm{mT} / \mathrm{m}$ ) gradients using single-shot spin echo, echo planar imaging (TE/TR: $68 / 5400 \mathrm{~ms} ; 1.2 \times 1.2 \times 1.2 \mathrm{~mm}^{3}$ voxels). The field of view was $220 \times 220$ $\mathrm{mm}$ in plane, the matrix size was $180 \times 180 \times 90$. Total scan time: 30 minutes and 25 seconds.

MICRA: Images were acquired on a 3.0T Siemens Connectom system with ultra-strong ( $300 \mathrm{mT} / \mathrm{m}$ ) gradients using single-shot spin echo, echo planar imaging (TE/TR $=59 / 3000 \mathrm{~ms}$; voxel size $=2 \times 2 \times 2 \mathrm{~mm}^{3}$ ). The field of view was $220 \times$ $220 \mathrm{~mm}$ in plane, the matrix size was $110 \times 110 \times 66$. Total scan time: 16 minutes and 37 seconds.

CNV: Images were acquired using a Siemens 3T Connectom MRI scanner with 14 b0 images, 30 directions at b = 500, $1200 \mathrm{~s} / \mathrm{mm}^{2}, 60$ directions at $b=2400,4000,6000 \mathrm{~s} / \mathrm{mm}^{2}$ (Delta/delta: $24 / 7 \mathrm{~ms}$ ). No cardiac gating.

cdMRI: Images were acquired on a Siemens 3T Connectom MRI scanner with 60 directions at $b=1200,3000$ and $5000 \mathrm{~s} / \mathrm{mm}^{2}$ (Delta/ delta: $31.1 / 8.5 \mathrm{~ms})$. No cardiac gating.

MICRA: Images were acquired on a 3.0T Siemens Connectom system with ultra-strong $(300 \mathrm{mT} / \mathrm{m})$ gradients. TE/TR $=59 / 3000 \mathrm{~ms}$; bvalues= 0 (14 vols), 200;500 (20 dirs), 1200 (30 dirs), and 2400;4000;6000(60 dirs) s/mm²). No cardiac gating.

\section{Preprocessing}

Preprocessing software

Normalization

Normalization template

Noise and artifact removal

Volume censoring
MRtrix (3.0.2) is available at www.mrtrix.org

FSL (5.0.9) is available at fsl.fmrib.ox.ac.uk/fsl/fslwiki.

SCILPY (1.0.0) is available at github.com/scilus/scilpy.

TractSeg (v2.2) is available at github.com/MIC-DKFZ/TractSeg.

Not applicable - Tractometry is performed in each subject's individual streamline space to mitigate the effect of registration artefacts. Points are matched between subjects using along-tract profiling.

Not applicable - see above.

Each dataset was denoised (Veraart et al. 2016), and corrected for signal drift (Vos et al. 2017), motion and distortion (Andersson et al. 2003, 2016), gradient non-linearities (Connectom data only, Glasser et al. 2013), and Gibbs ringing (Kellner et al. 2016).

Slice-wise outlier detection was performed using SOLID (Sairanen et al. 2018)

\section{Statistical modeling \& inference}

Model type and settings

3 anomaly scores were derived for each subject using 1) univariate $z$-score, 2) multivariate Mahalanobis distance based on Principal Component Analysis (PCA) and 3) Mean Absolute Error (MAE), i.e., the reconstruction error of an Autoencoder neural network.

Effect(s) tested

2 tailed t-test assuming equal variance between the AUC scores of all 3 methods.

Specify type of analysis:

Whole brain

Anatomical location(s)
Major white et al. 2018)
Statistic type for inference (See Eklund et al. 2016)

Correction
-Point-wise inference along the tracts.

-2-tailed t-tests assuming equal variances between the AUC scores.

Data permutations:

Single subject analysis: Leave-one-out-cross-validation (LOOCV).

CNV and CNP: Training was done using healthy controls data only (80\%). Evaluation was done using a random matching subset of healthy controls and patients (20\%) repeated 100 times to assess variations in the model.

Multiple comparisons for the AUC bar plots:

Bonferroni at alpha $=0.01$ ( 3 methods $\times 4$ metrics $=12$ comparions, $\mathrm{p}<8.3 \mathrm{e}-4)$ 
\ Multivariate modeling or predictive analysis sizes from each groups. Specifically, the autoencoder is trained using healthy controls data only. Therefore, the entire dataset is first split into a training set (80\%, made of healthy controls only) and a validation set ( $20 \%$, by combining the patients with a matching number of healthy controls). $10 \%$ of the training set is held out for testing during the training phase to evaluate the loss. Age and sex regression and feature normalization (min-max) were performed on the normative training set and subsequently applied to the held-out validation set subsequently to prevent information leakage. To derive conservative estimates and assess variations within the model, we repeated this process 100 times and report the mean MAE for each subject. Finally, we compared the sensitivity versus specificity of the anomaly scores using the mean receiver operating characteristic (ROC) area under the curve (AUC) across iterations, and standard deviations used as uncertainties. Comparisons AUC scores between patients and controls were performed with t-tests for the balanced groups and Kolmogorov-Smirnov (KS) test for the unbalanced groups. The correlation between anomaly scores and clinical scores was computed using the Spearman's rho.

Autoencoder: Our autoencoder implementation consists of a symmetric design of five fully connected layers (In). The input and output layers (II and I5) have exactly the same number of nodes as the number of input tracts features. The inner layers (12 and 14) consecutively apply a compression ratio of 2 by reducing the number of nodes by half, up to the bottleneck hidden layer (I3). For example, if using an input vector made of 100 features, $I 1$ and 15 will consist of 100 nodes, 12 and 1450 nodes, and 25 nodes for the bottleneck 13 . Rectified linear units (ReLU) activation were used between the layers to promote sparse activation and tanhfor the last layer (epochs: 25 , batch size $=24$, learning rate: $1.0 e-3$, optimiser: Adam, loss: mean squared error, validation split: 0.1 ). To promote sparsity and reduce overfitting, an activation penalty was imposed to the bottleneck layer using I1-regularization (10e-5). The mean absolute error (MAE) was used as anomaly score.

Z-score: Z-scores were computed for each tract segment and averaged to derive a subject-specific anomaly score at each iteration (described above). The mean over all iterations was retained as anomaly scores for all subjects.

PCA: Principal Component Analysis (PCA) was applied to the set of features by restricting the dimensionality to $85 \%$ of explained variance. Next, the Mahalanobis Distance was used to derive an anomaly score. The problem of anomaly detection can be seen as a one-class classification problem and therefore, our training data only contains healthy controls. 